\title{
Síntesis y almacenamiento de carga de la nueva aleación polipirrol/poli(ácido maleico-olefina)
}

\author{
F.D'ERAMO' 1 , T.F.OTERO ${ }^{2}$ \\ ${ }^{1}$ Universidad Nacional de Río Cuarto, Facultad de Ciencias Exactas Físico, Químicas y Naturales, Depto. de Química y Física, Estafeta № 9 , 5800 \\ Río Cuarto, Argentina. E-Mail: fderamo@arnet.com.ar \\ ${ }^{2}$ Universidad del País Vasco, Facultad de Química, Laboratorio de Electroquímica, Apartado 1072, 20018 San Sebastián, España
}

\begin{abstract}
Se ha analizado la influencia de diversas variables de síntesis sobre la capacidad de almacenamiento de carga (mC de carga almacenada / mg de polímero electrogenerado) de aleaciones poliméricas de polipirrol/poli(ácido maléico-co-olefina), como paso inicial para la optimación de este material para su aplicación en baterías todo orgánicas. Las variables de estudio han sido: el potencial y el tiempo de polimerización, las concentraciones de pirrol y poli(ácido maléico-co-olefina) y la temperatura de la solución de polimerización. Optimando estos parámetros se ha logrado sintetizar materiales con una capacidad promedio de almacenamiento de carga de $120 \mathrm{mC} . \mathrm{mg}^{-1}$.
\end{abstract}

Palabras claves: polipirrol, poli(ácido maléico-co-olefina), sintesis

Synthesis and charge storage ability of the new polyrrole/poly (maleic acid-co-olefin) blend

The influence that the electrosynthesis conditions of the polypyrrole/poly(maleic acid-co-olefin) blend have on the charge storage ability (amount of electrical charge stored $(\mathrm{mC})$ per unit mass $(\mathrm{mg}$ ) of electrogenerated polymer) has been analysed. This is the initial step for the optimisation, pyrrole and poly (maleic acid-co-olefin) concentrations and the polymerisation temperature have been the studied parameters. Optimising these ones, we could obtain materials with average storage charge of $120 \mathrm{mc}^{\mathrm{mg}}{ }^{-1}$

Keywodrs: polypyrrole, poly (maleic acid-co-olefin), synthesis

\section{INTRODUCCIÓN}

Durante los últimos años, el empleo de los polímeros conductores en la construcción de baterías poliméricas concitó el interés, en vista de las múltiples ventajas que representa su uso (1). En estas baterías, se utilizan polímeros conductores del tipo-p y tipo-n, como cátodo y ánodo, respectivamente (2). En general, los polímeros conductores que se emplean como ánodo poseen una baja estabilidad y durabilidad, lo que ha llevado a que sean los electrodos metálicos, como los de Li, o de polímeros neutros (que se preparan reduciendo un polímero conductor del tipo-p) los que se utilicen con mayor frecuencia.

Hoy en día los esfuerzos están dirigidos hacia el desarrollo de nuevos materiales poliméricos que puedan desempeñar esta función, dada las claras ventajas que poseen la baterías "todo orgánicas" frente a las convencionales, como es su alta velocidad de carga y descarga, lo que permite obtener grandes potencias eléctricas. Una alternativa podría ser el empleo de las denominadas aleaciones poliméricas, como por ejemplo, las que se obtienen al electropolimerizar un monómero, como pirrol, en presencia de un polielectrolito (polianión). La incorporación de este polielectrolito dentro de la matriz del polímero conductor tiene una marcada influencia en las propiedades morfológicas, ópticas y electroquímicas de los materiales sintetizados. Estas variaciones dan lugar al uso de estos nuevos materiales en diferentes campos tecnológicos, entre ellos, su empleo como sensores (3) ó como ánodo en baterías orgánicas $(4,5)$. Respecto a esta última aplicación, una propiedad de vital importancia para su aplicación en baterías es la "capacidad de almacenamiento de carga" que se define como la relación entre la carga almacenada en la aleación polimérica y su masa.

Por otra parte, es ampliamente conocido que las propiedades físicas, químicas y mecánicas de un nuevo material polimérico y por lo tanto su aplicabilidad, están íntimamente relacionadas con las condiciones de síntesis, por lo que para lograr un buen control sobre las propiedades de un determinado material es necesario tener un adecuado conocimiento de las variables que influyen durante su síntesis. El objetivo final de este tipo de estudios es obtener materiales con mejores propiedades.

Siguiendo esta línea de razonamiento, el presente trabajo se centra en la síntesis y análisis de la influencia que ejercen ciertas variables del medio sobre la capacidad de almacenar carga de una nueva aleación polimérica de: polipirrol/ poli(ácido maléico-co-olefina) (poli(Pi/PAM-co-O)). Este estudio es el paso inicial para optimizar este nuevo material en vista de su posible aplicación como ánodo en baterías orgánicas.

\section{PROCEDIMIENTO EXPERIMENTAL}

Las soluciones para obtener las aleaciones poliméricas de poli(Pi/PAM-co-O) fueron preparadas a partir de pirrol en una solución acuosa de PAM-co-O. En la preparación de estas soluciones se empleó pirrol marca Janssen, purificado previamente por destilación a vacío $\left(59^{\circ} \mathrm{C}, 50\right.$ Torr $)$ y PAM- 
co-O Aldrich con un contenido de $\mathrm{Na}$ del 3\%, de calidad p.a. Todas las concentraciones de polielectrolito de este trabajo estan referidas al contenido de $\mathrm{Na}$. Las disoluciones de trabajo fueron desoxigenadas mediante burbujeo con nitrógeno durante cinco minutos antes de iniciar la electrosíntesis. Esto evita la oxidación prematura del monómero, lo cual puede introducir contaminantes en el material durante la electropolimerización. Agua triplemente destilada procedente de un sistema Milli-Q-Reagent fue empleada como disolvente.

Las experiencias electroquímicas se llevaron a cabo en una celda, de un solo compartimento, herméticamente cerrada para evitar la interferencia del oxígeno. Como electrodo de trabajo se empleó una lámina de platino $(\mathrm{Pt})$ de $1 \mathrm{~cm}^{2}$ y como contraelectrodo otra lámina de Pt de $2 \mathrm{~cm}^{2}$ de superficie. Un electrodo de $\mathrm{Ag} / \mathrm{Ag}^{+}$fue utilizado como electrodo de referencia. El método electroquímico empleado para generar las aleaciones poliméricas fue el de saltos de potencial. Se empleó para ello un potenciostato/galvanostato PAR 273 conectado a un microprocesador IBM PS y controlado desde él mediante un programa M270 de EC\&G.

Una vez generados los materiales, éstos se enjuagan con el disolvente, se secan y se pesan empleando una ultramicrobalanza SARTORIUS 4504 MP8 de $10^{-7}$ gr. En aquellas experiencias en las cuales la temperatura fue la variable de estudio, se empleó un baño criostático HUBER MINISTAT para su control.

\section{RESULTADOS Y DISCUSIÓN}

\subsection{Preparación de poli(Pi/PAM-co-O)}

El electrodo base (Pt) inmerso en la solución del monómero, se somete a un salto de potencial, como el que se observa en la Fig.1a. El salto se realiza desde un potencial en el que no hay reacción electródica $(0 \mathrm{mV})$ hasta un potencial más anódico (potencial de polimerización, Ep) que el de oxidación del monómero (próxima a $500 \mathrm{mV}$ ). Este potencial se mantiene por un tiempo determinado (tiempo de polimerización, tp). En los estudios en donde el Ep es la variable de estudio, el límite superior de potencial está dado por la oxidación del polielectrolito o del disolvente (próxima a 2000 $\mathrm{mV}$ en este sistema). Por lo tanto, el intervalo de potencial empleado en este trabajo está comprendido entre $500 \mathrm{mV}$ y $2000 \mathrm{mV}$. Mientras se mantiene el electrodo de trabajo al potencial catódico no se observa ningún paso neto de corriente. Al realizar el correspondiente salto de potencial hasta el valor anódico, se observa en todas las condiciones estudiadas, paso de corriente con el progresivo oscurecimiento del electrodo de Pt con el tiempo, como consecuencia del depósito de la aleación polimérica sobre el mismo.

Una vez depositado el material sobre el electrodo de Pt, el mismo se enjuaga, se seca y se pesa. La masa de aleación polimérica $(\mathrm{mp})$ electrodepositada se obtiene por diferencia de peso con el electrodo de Pt limpio.

Asimismo, diferentes tp permiten obtener materiales de espesores diferentes. La correlación entre la masa de material electrogenerado y el tiempo invertido en su obtención se muestran en la Fig.1b, como se puede observar ésta es lineal. Este resultado indica que dicho material crece uniformemente sobre la superficie del electrodo a medida que aumenta el tiempo de polimerización. Dicha experiencia nos revela que, mediante éste método de síntesis, es posible controlar cuantitativamente la reacción de polimerización. (a)
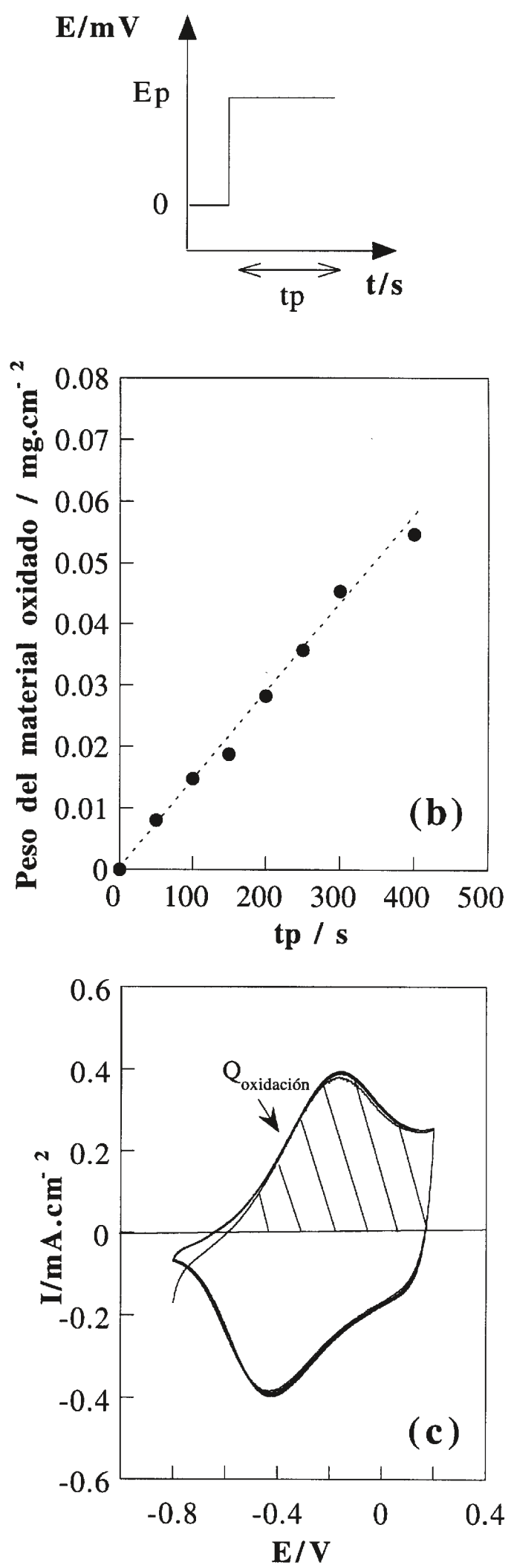

Figura 1: (a) Perfil de la onda E-t empleada en la electrogeneración de poli(Pi/PAM-co-O). (b) Variación de la masa de material oxidado con el tp. Condiciones de síntesis: solución acuosa 0,35 M Pi +9x10-4 M PAM-co-O; $\mathrm{Ep}=1200 \mathrm{mV} ; \mathrm{T}=20^{\circ} \mathrm{C}$. (c) Voltamperograma de control del material en $0,1 \mathrm{M} \mathrm{LiClO}_{4} / \mathrm{H} 2 \mathrm{O}$ a $10 \mathrm{mV}^{-1}$. 
Siguiendo este procedimiento de síntesis para electrogenerar las aleaciones poliméricas y recurriendo a la vasta experiencia de nuestro grupo en la optimación de propiedades de materiales poliméricos de diversas características (6-11), podemos establecer las variables físicas, eléctricas y químicas, que pueden afectar de manera directa esta propiedad del material en estudio. Así, en este trabajo analizaremos la influencia del Ep, tp, la concentración de pirrol y de PAMco-O y la temperatura de la solución de generación.

\subsection{Control y comportamiento electroquímico de poli(Pi/ PAM-co-O)}

El electrodo recubierto con la aleación polimérica, siguiendo el procedimiento descrito en el punto anterior, se transfiere a la solución de control $\left(0,1 \mathrm{M} \mathrm{LiClO}_{4} / \mathrm{H}_{2} \mathrm{O}\right)$, libre de monómero. Una vez allí, el potencial se cicla potenciodinámicamente entre los límites $-600 \mathrm{mV}$ y $200 \mathrm{mV}$ (vs Ag/ $\mathrm{Ag}^{+}$) a una velocidad de barrido de $20 \mathrm{mV} . \mathrm{s}^{-1}$. El resultado de los barridos de potencial son los voltamperogramas, representaciones de la densidad de corriente frente al potencial, como el que se muestra en la Fig.1c. En dicha gráfica se puede observar que al barrer el potencial desde valores catódicos $(-600 \mathrm{mV})$ hacia valores anódicos, se produce un aumento en la densidad de corriente hasta que se define un pico (I, en Fig. 1b) centrado alrededor de $-200 \mathrm{mV}$. Al invertir el barrido de potencial aparece un nuevo pico, II, en aproximadamente $-400 \mathrm{mV}$. Este sistema de picos I/ II está asociado a la oxidación y reducción del material, respectivamente. Estos procesos rédox, continuos y reversibles, pueden ser visualizados físicamente en el siguiente esquema:

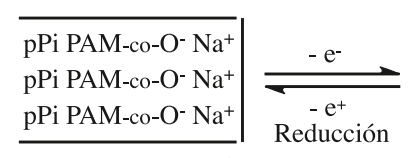

(sólido)

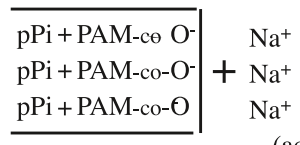

(sólido) (aq)
Desde el punto de vista del empleo de estos materiales como ánodo en baterías orgánicas, el proceso de reducción correspondería al cargado de la batería y el de oxidación al proceso de descarga del mismo.

Como se puede observar, durante la reducción de estos materiales, el polielectrolito queda atrapado dentro de la matriz polimérica, ya que sus cadenas se entrelazan con las de polipirrol. Para mantener el principio de electroneutralidad, el sistema incorpora cationes del medio que se asocian con el polianión $(12,13)$. Durante el proceso de oxidación, estos cationes se expulsan nuevamente a la solución y se generan a lo largo de la cadena de polipirrol cargas positivas. Dado que el proceso de oxidación es reversible, esta carga que queda almacenada en el seno del polímero puede recuperarse en el proceso de reducción.

Por otra parte, puesto que en las experiencias que se llevan a cabo en este trabajo estaremos manejando aleaciones poliméricas de masa diferente $y$, como consecuencia, de espesor diferente, el comportamiento electroquímico de estos materiales, generados a distintos Ep ó tp, no puede ser directamente comparado. Por lo tanto, resulta imprescindible definir una magnitud relativa relacionada con el comportamiento electroquímico del material pero que sea independiente de la masa o espesor del mismo. Surge entonces el concepto de "capacidad de almacenamiento de carga" definida como los $\mathrm{mC}$ de carga almacenada por el material por
TABLA I. EVOluCiÓN DE LA CAPACIDAD DE ALMACENAMIENTO DE CARGA DE POLI(PI/PAM-CO-O) CON EL POTENCIAL DE POLIMERIZACIÓN

\begin{tabular}{|c|c|}
\hline Ep/mV & $\begin{array}{c}\text { Capacidad de } \\
\text { Almacenamiento } \\
\text { de carga (mC.mg-1 }\end{array}$ \\
\hline 800 & 119,3 \\
\hline 900 & 120,5 \\
\hline 1000 & 119,3 \\
\hline 1200 & 124,6 \\
\hline 1400 & 121,4 \\
\hline 1600 & 115,4 \\
\hline 1800 & 94,3 \\
\hline 2000 & 83,2 \\
\hline
\end{tabular}

mg de polímero electrogenerado. La carga almacenada en el proceso de oxidación $\left(\mathrm{Q}_{\text {oxidación }}\right)$ o la que se recupera al reducir la aleación polimérica, se obtienen de la integración de la curva voltamperométrica en la solución de control (Fig.1c).

\subsection{Influencia de las variables de síntesis sobre la capaci- dad de almacenamiento de carga de poli(Pi/PAM- co-O)}

El objetivo de estas experiencias es establecer el intervalo más adecuado para cada una de las variables de electrosíntesis del sistema, condición que será aplicada posteriormente en el estudio de la variable siguiente.

En primer lugar se analizó la influencia del Ep sobre la capacidad de almacenar carga de poli(Pi/PAM-co-O). Para estas experiencias se preparan distintas aleaciones poliméricas, variando el Ep y manteniendo constantes las demás variables. Los mismas se generan en disolución acuosa 0,35 M Pi y $5 \times 10^{-4}$ M PAM-co-O, a temperatura ambiente, aplicando el potencial en estudio durante $250 \mathrm{~s}$. Luego, se calcula la capacidad de almacenamiento de carga tal como se mencionó anteriormente. La correlación entre dicha propiedad y el Ep empleado para la generación de cada material se muestra en la Tabla I. Para películas sintetizadas a potenciales inferiores a $1600 \mathrm{mV}$, la capacidad del material para almacenar carga permanece prácticamente constante. Para materiales generados a Ep superiores a $1600 \mathrm{mV}$ se produce una clara disminución de la misma. Este comportamiento debe estar motivado por la presencia de reacciones paralelas al proceso de polimerización, cuya contribución relativa varía al modificar el Ep. Dichas reacciones competitivas pueden provocar la degradación del material haciéndolo menos susceptible a la oxidación y repercutiendo, por lo tanto, negativamente en la capacidad de carga y descarga de los mismos.

Teniendo en cuenta estos resultados podemos establecer que con el objetivo de conseguir el mejor rendimiento en lo referido a la capacidad para almacenar carga de los materiales resultantes, la síntesis de polipirrol desde soluciones acuosas de PAM-co-O debe realizarse a potenciales comprendidos en el intervalo de $800 \mathrm{mV}$ a $1600 \mathrm{mV}$. Para continuar con los estudios se eligió un potencial intermedio de $1200 \mathrm{mV}$ para generar las aleaciones poliméricas. 
Posteriormente, se electrogeneraron materiales a distintos tiempos de polimerización y para distintas concentraciones de pirrol. El intervalo de tiempos de polimerización estudiado fue de 50 a 400s para cada una de las concentraciones de monómero ensayadas: 0,05 M; 0,1 M; 0,2 M; 0,35 M y 1M. La concentración de PAM-co-O fue en todos los casos 5x10-4 M, el Ep $1200 \mathrm{mV}$ y la temperatura $20^{\circ} \mathrm{C}$. La Tabla II muestra la variación de la capacidad de almacenamiento de carga de todas las aleaciones poliméricas sintetizadas en las condiciones mencionadas.

En su evolución con el tiempo, esta propiedad muestra, en todos los casos, una tendencia a aumentar a medida que tp se incrementa, tomando valores prácticamente constantes para tiempos superiores a $200 \mathrm{~s}$. Esto significaría que, para unas condiciones iniciales de síntesis dadas, la capacidad rédox de los materiales aumenta con el tiempo de generación. Así, para la mayoría de las concentraciones de monómero estudiadas, la capacidad de almacenamiento de carga entre los 50 y $400 \mathrm{~s}$ de polimerización aumenta entre un 25 a $35 \%$. Un comportamiento similar se encontró al analizar esta propiedad en materiales obtenidos a diferentes concentraciones de Pi. Se pudo observar un aumento continuo de esta propiedad para concentraciones de monómero inferiores a $0,2 \mathrm{M}$. A concentraciones superiores, y para todos los tp estudiados, se produce una estabilización de la misma.

De acuerdo con estos resultados, podemos establecer que las máximas capacidades de almacenamiento de carga para estos materiales se consiguen bajo las siguientes condiciones, en cuanto a las dos variables analizadas: tp superiores a $200 \mathrm{~s}$ y concentraciones de Pi mayores que 0,2 M. Por lo tanto, en estudios posteriores se emplearon tp de $250 \mathrm{~s}$ (por razones prácticas) y soluciones acuosas de $0,35 \mathrm{M}$ de $\mathrm{Pi}$.

Siguiendo la metodología descrita, se investigó el efecto de la concentración de PAM-co-O. Para llevar a cabo estas experiencias se emplearon disoluciones de $\mathrm{Pi}$ 0,35 $\mathrm{M}$ con concentraciones de polielectrolito en el intervalo $1 \times 10^{-4} \mathrm{M}-3 \times 10^{-3} \mathrm{M}$. El Ep fue $1200 \mathrm{mV}$, el tp de $250 \mathrm{~s}$ y la temperatura de $20^{\circ} \mathrm{C}$. Los valores correspondientes a las capacidades de almacenamiento de carga, se encuentran representados en la Fig.2. Como se puede observar, dicha propiedad disminuye gradualmente al aumentar la concentración del polielectrolito. Esta pérdida de la capacidad rédox del material podría atribuirse a un cambio en la composición del mismo al aumentar la concentración de PAM-co-O. Estudios realizados con otros polielectrolitos en nuestro laboratorio (11,14-19) han mostrado que, este tipo de compuestos, se adsorbe tanto sobre la superficie del electrodo base como en la matriz del polímero durante su generación. Al aumentar la concentración de polielectrolito en la solución probablemente se incrementa su velocidad de adsorción, como consecuencia, los materiales que se sintetizan en soluciones concentradas de PAM-co-O tendrán un mayor contenido de polielectrolito en su matriz. Puesto que PAM-co-O sólo contribuye con su masa al material, dado que es electroinactivo en la ventana de potencial de trabajo, estaremos generando materiales de mayor masa pero con menor capacidad de carga y descarga.

Para seleccionar el intervalo de concentración de PAMco-O óptimo, según el objetivo planteado en este trabajo, nos encontramos con una situación de compromiso: bajas concentraciones de polielectrolito dan materiales con mayor capacidad para almacenar carga, sin embargo, la conductividad de la solución es el factor limitante. Por lo tanto, se seleccionó como intervalo de concentración de PAM-co-O más apropiado para sintetizar estos materiales el comprendido entre $5 \times 10^{-4} \mathrm{M}$ y $1 \times 10^{-3} \mathrm{M}$.

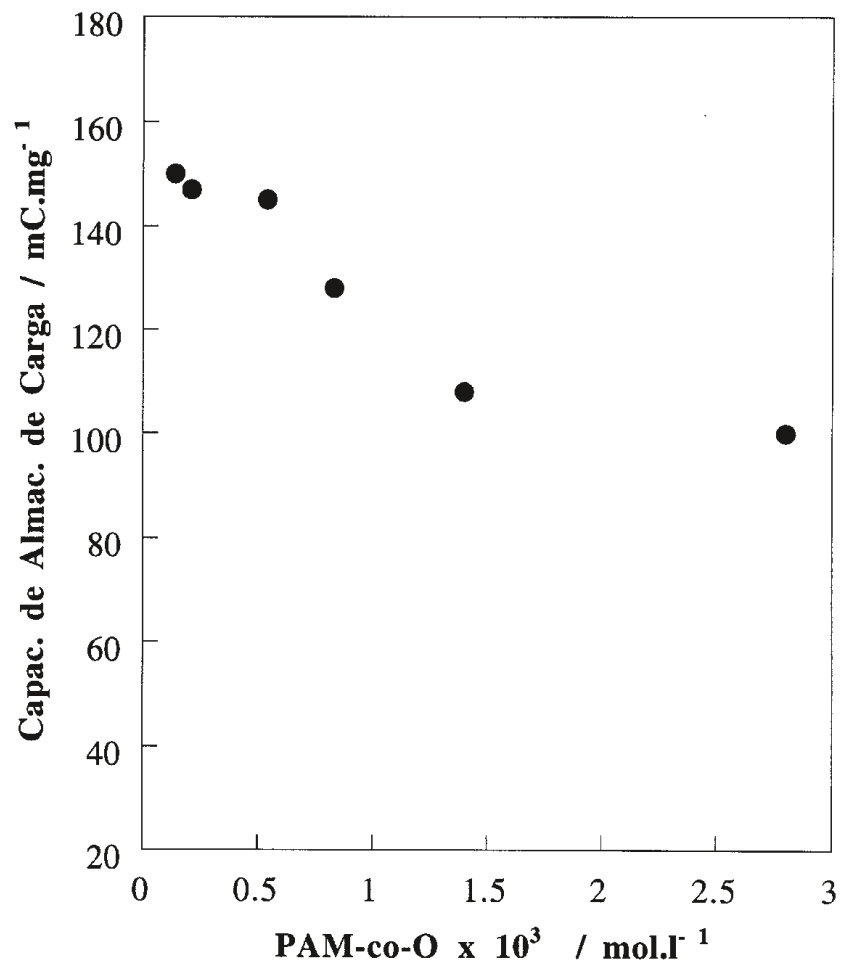

Figura 2: Evolución de la capacidad de almacenamiento de carga de poli(Pi/ PAM-co-O) con la concentración de PAM-co-O.

TABla II. Evolución DE LA CAPACIDAD DE ALMACENAMIENTO DE CARGA DE POLI(PI/ PAM-CO-O) CON EL TIEMPO DE POLIMERIZACIÓN, PARA DIFERENTES CONCENTRACIONES DE MONÓMERO

\begin{tabular}{|c|c|c|c|c|c|}
\hline & \multicolumn{5}{|c|}{ Concentración de Pi / mol.. $\mathbf{1}^{\mathbf{1}}$} \\
\hline tp & $\mathbf{0 , 0 5} \mathbf{M}$ & $\mathbf{0 , 1} \mathbf{M}$ & $\mathbf{0 , 2} \mathbf{M}$ & $\mathbf{0 , 3 5} \mathbf{M}$ & $\mathbf{1} \mathbf{~}$ \\
\hline 50 & 48,7 & 72,8 & 79,2 & 97,1 & 100,9 \\
\hline 100 & 49,8 & 79,9 & 83,4 & 108,2 & 112,8 \\
\hline 150 & 52,3 & 80,3 & 94,6 & 114,6 & 127,7 \\
\hline 200 & 56,2 & 84,3 & 115,3 & 120,8 & 135,4 \\
\hline 250 & 58,4 & 92,5 & 125,6 & 135,6 & 139,7 \\
\hline 300 & 62,3 & 90,6 & 127,4 & 132,4 & 140,6 \\
\hline 400 & 65,1 & 91,8 & 130,1 & 129,7 & 138,5 \\
\hline
\end{tabular}

Para finalizar con este estudio sistemático de la influencia de las condiciones de síntesis sobre la capacidad de almacenar carga de estos nuevos materiales, se analizó el efecto de la temperatura de la solución de electrogeneración. En este caso, las aleaciones poliméricas se sintetizan a partir de soluciones acuosas $0,35 \mathrm{M}$ Pi y $7 \times 10^{-4} \mathrm{M}$ PAM-co-O, por polarización del electrodo de trabajo a $1200 \mathrm{mV}$ durante 250s. El intervalo de temperatura estudiado fue de $5{ }^{\circ} \mathrm{C}$ a $45^{\circ} \mathrm{C}$. La Fig. 3 resalta la influencia de esta variable sobre la capacidad de almacenamiento de carga de carga de los materiales sintetizados. Hasta los $20^{\circ} \mathrm{C}$, esta propiedad, aunque decreciente, se mantiene en valores bastante similares. Sin embargo, las películas sintetizadas a $30^{\circ} \mathrm{C}$ y $45{ }^{\circ} \mathrm{C}$ presentan capacidades de almacenamiento de carga inferiores a un $32 \%$ a las 


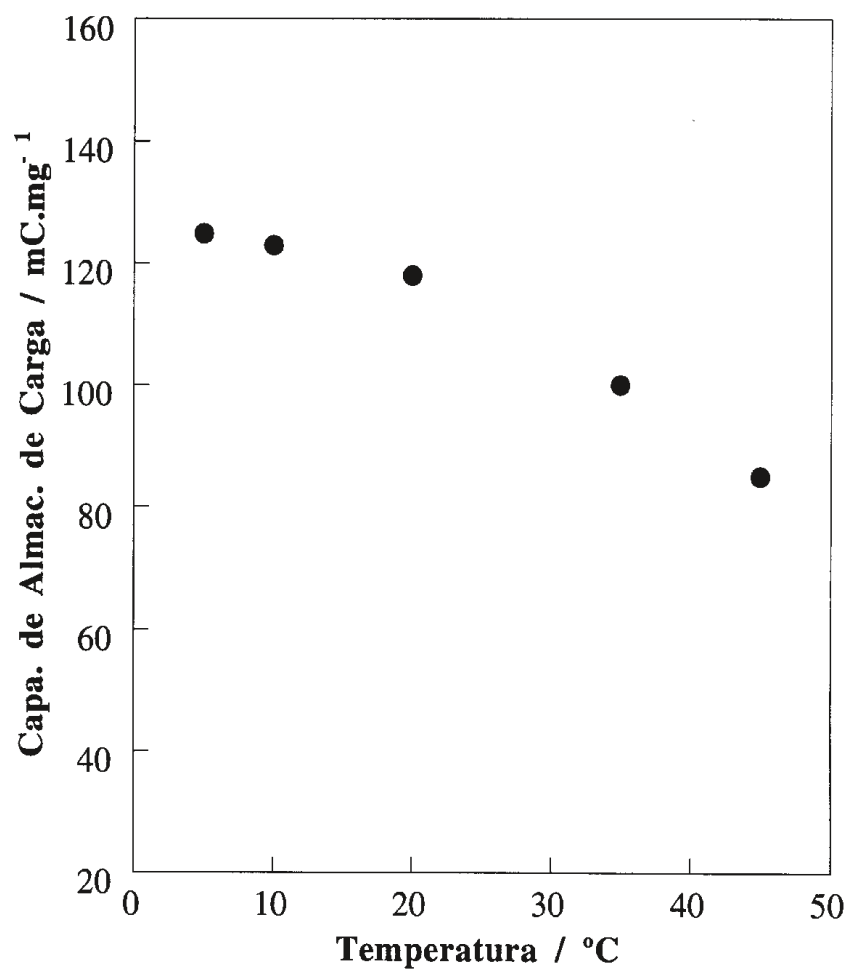

Figura 3: Evolución de la capacidad de almacenamiento de carga de poli(Pi/ PAM-co-O) con la temperatura de la solución de electrogeneración.

obtenidas con materiales generados a $5{ }^{\circ} \mathrm{C}$. La pérdida de la capacidad rédox de las aleaciones poliméricas al aumentar la temperatura de polimerización apuntaría nuevamente hacia la existencia de procesos competitivos que alteran la naturaleza del material, degradándolo. A partir de estos resultados se pudo establecer, que la síntesis de estos materiales a temperatura ambiente (aproximadamente, $18{ }^{\circ} \mathrm{C}$ ó $20^{\circ} \mathrm{C}$ ) sería adecuado, puesto que el aumento de la capacidad de almacenamiento de carga a $5{ }^{\circ} \mathrm{C}$ no es lo suficientemente significativo como para justificar el montaje de un sistema más complejo (baño termostático, celda con camisa de refrigeración), para generar estos materiales.

\section{4.- CONCLUSIONES}

Los resultados presentados en este trabajo muestran claramente que las condiciones de síntesis tienen una marcada influencia sobre la capacidad de almacenamiento de carga de las aleaciones poliméricas de poli(Pi/PAM-co-O). Se pudo observar, la baja influencia del potencial de polimerización sobre esta propiedad, lo cual muestra una clara ventaja de estos materiales, desde un punto de vista tecnológico. Asimismo, un incremento, tanto del tiempo de polimerización, como de la concentración de pirrol, provocan un aumento en la capacidad de almacenar carga, hasta alcanzar un valor constante y próxima a $120 \mathrm{mC} \cdot \mathrm{mg}^{-1}$. Sin embargo, se pudo comprobar que un aumento de la concentración de PAM-co-O, produce un efecto totalmente opuesto sobre esta propiedad: la misma disminuye gradualmente al incrementar la concentración del polielectrolito.

En conclusión, ha quedado demostrado en este trabajo la importancia de controlar las variables de síntesis para optimizar una propiedad de un determinado material. Así, con este estudio sistemático hemos podido establecer cuales son las mejores condiciones de síntesis para poli(Pi/PAM-co-O), para generar materiales con su máxima capacidad de almacenamiento de carga.

\section{AGRADECIMIENTOS}

Los autores desean expresar su agradecimiento a la Universidad Nacional de Río Cuarto, Córdoba, Argentina, a la Diputación Foral de Gipúzkoa, al Gobierno Vasco y a CEGASA por su apoyo económico.

\section{BIBLIOGRAFÍA}

1. C. Arbizzani, M. Mastragostino, B.Scrosati, “Conducting polymers for batteries, supercapacitors and optical devices", Handbook of Organic Conductive Molecules and Polymers, Hari Singh nalwa, ed. vol 4, capitulo 11, pag.595-619. John Wiley \& Sons, Chichester (1997).

2. D. MacInnes, Jr., M.A. Druy, P.J. Nigrey, D.P. Nairns, A.G.MacDiarmid and A.J.Hegger, "Organic Batteries: Reversible n- and p-Type Electrochemical Doping of Polyacetylene, $(\mathrm{CH})_{x}{ }^{\prime \prime}$, J.Chem.Soc., Chem. Commun, 317-319 (1981).

3. R.C. Martínez, F.B. Domínguez, F.M. González, J.H. Méndez and R.C. Orellana, "Polypyrrole-dodecyl sulphate electrode as a microsensor for electroinactive cations in flow-injection analysis and ion chromatography", Anal. Chim. Acta, 279 299-307 (1993).

4. T. Shimidzu, A. Ohtani, T. Iyoda and K. Honda, “A Novel Type of Polymer Battery using a Polypyrrole-Polyanion Blend Anode", J. Chem. Soc., Chem. Commun., 327-328 (1987).

5. T. Osaka, T. Momma and K. Nishimura, “Electroactivity Change of Electropolymerized Polypyrrole/ Polystyrenesulfonate Blend Film in Some Organic Electrolytes", Chem. Lett., 1787-1790 (1992).

6. T.F. Otero and E. de Larreta-Azelain, "Electrochemical control of the morphology, adherence, appearance and growth of polypyrrole films", Synth. Metals, 26 79-88 (1988).

7. T.F. Otero, J. Rodríguez and E. de Larreta-Azelain, “Electrogeneration of polythiophene films. Temperature influence on morphologic and physic properties", Polymer, 31 220-222 (1990).

8. T.F. Otero and C. Santamaría, "Redox behaviour of thin polypyrrole films. Optimization of response times", Solid State Ionics, 63 810-815 (1993).

9. T.F. Otero, C. Santamaría and J. Rodríguez, "Optimization of conducting polymers synthesis for battery applications", Materials Research Society Symp. Proc., 328 805-810 (1994).

10. T.F. Otero and J. Rodríguez, "Parallel kinetic studies of the electrogeneration of conducting polymers: mixed materials, composition and properties control", Electrochim. Acta, 39 245-253 (1994).

11. T.F. Otero and J.M. Sansiñena, "Influence of synthesis conditions on polypyrrole-poly(styrenesulphonate) composite electroactivity", J. Electroanal. Chem., 412 109-116 (1996).

12. F. Li and w.J. Albery, "Electrochemical and in situ electron paramagnetic resonance studies of Polypyrrole doped with Polystyrene Sulphonate", J. Chem. Soc, Faraday Trans., 87 2949-2954 (1991).

13. M. Lien, W.H. Smyrl and M. Morita, "Cation and anion insertion in separate processes in poly(pyrrole) blend film", J. Electroanal. Chem., $309333-$ 340 (1991).

14. T.F. Otero and M.V. Vázquez, "Electrogeneration of a blend polypyrrolecarboxymethylcellulose: kinetic study", J. Electroanal. Chem., 397 171-176 (1995).

15. T.F. Otero and V. Olazabal, "Electrogeneration of polypyrrole in presence of polyvinylsulphonate. Kinetic study", Electrochim. Acta, 41 213-220 (1996).

16. T.F. Otero and M.J. González-Tejera, "Polypyrrole + polyacrylate composites: electrogeneration", J. Electroanal. Chem., 410 69-77 (1996).

17. T.F. Otero and M.J. González-Tejera, "Polypyrrole + polyacrylate composites: kinetic study", J. Electroanal. Chem., 429 19-25 (1997).

18. T.F. Otero, P. Herrasti, P. Ocón and C.R. Alves, " Electrogeneration of polypyrrole- carboxymethylcellulose composites: electrochemical, microgravimetric and morphological studies", Electrochim. Acta, 43 1089-1100 (1998).

19. T.F. Otero and O. Roig, "Polypyrrole-Poly(styrene-alt-maleic acid) blend: electrogeneration and kinetic study for a tailored synthesis", enviado a publicar al J. Electrochem. Soc. 